\title{
ESTUDIO DE LA CIMENTACIÓN DE LOS PILARES DE LA CATEDRAL DE SEVILLA
}

\author{
( STUDY OF THE FOUNDATIONS OF THE PILLARS OF SEVILLE'S CATHEDRAL)
}

\author{
Ángela Barrios Padura, Arquitecta; Francisco Borja Barrera, Dr. en Geografia \\ e Ignacio Valverde Espinosa, Dr. en Geología \\ Ángel Martínez Girón y Jorge Polo Velasco (VORSEVI, S.A.) \\ Alfonso Jiménez Martín, Catedrático de Universidad y Jesús Barrios Sevilla, Catedrático de Universidad \\ (E.T.S. de Arquitectura de Sevilla)
}

ESPAÑA

Fecha de recepción: 9-II-97

\section{RESUMEN}

Se realiza el presente estudio atendiendo a una doble demanda. De un lado, conocer la tipología de la cimentación $y$ del terreno de apoyo de los pilares de la catedral de Sevilla $y$, de otro, determinar si las fisuras que presentan las mismas tienen un origen geotécnico. La investigación realizada ha permitido conocer que la cimentación tiene un sobreancho de $1 m$ en relación con la sección del pilar y una profundidad de 5,70 - 5,90 m. En cuanto al terreno de apoyo, se han comprobado sus caracteristicas geotécnicas, deduciéndose que el largo periodo de construcción de los pilares, bóvedas y cubiertas (90 años) permitió una lenta consolidación y drenaje del terreno, lo que evitó posibles desplomes o caidas. Esta conclusión determina la continuación de la investigación que se centrará, ahora, en el estudio de las caracteristicas de los pilares y de las posibles causas que han podido propiciar la aparición de fisuras.

Desde el punto de vista de la geoarqueología se han obtenido datos importantes sobre el nivel ocupacional de esta zona. La amplia investigación realizada ha permitido extraer importantes conclusiones sobre la secuencia de ocupación de esta zona desde 1.000 años a.C.

\section{SUMMARY}

This study is done in order to answer a double request. On one hand to know the tipology of foundation and the tipology of the bearing soil of pillars of the cathedral in Seville, and on the other hand to determine if the existing cracks have a geotechnical origin. The accomplished research let us know that foundation is $1 \mathrm{~m}$ overwide relative to the column section, and $5.70-5.90 \mathrm{~m}$ deep. As regards bearing soil, its geotechnical characteristics have been proved, so it may be deduced that the long period (90 years) for the construction of pillars, vaults and decks, allowed a slow consolidation and drainage of soil that have avoided a possible collapse or fall. According to this conclusion, the research will continue focusing now on the study of the characteristics of pillars and on the possibles reasons that have provoked the appearance of fissures.

From a geoarchaeological point of view, significant data of the occupational level of this area have been obtained. There are important conclusions to be drawn from the accomplished research about the sequence of occupation of this area since 1.000 years a.C.

\section{Introducción}

Lagran extensión de la catedral de Sevilla, unos $24.000 \mathrm{~m}^{2}$, que la sitúan como la mayor catedral gótica y la tercera iglesia del mundo, después de San Pedro de Roma y San Pablo de Londres, y la variedad de construcciones que la configuran, hacen que sea una fuente inagotable de investigaciones de todo tipo. Este trabajo es uno más, formando parte de la Tesis Doctoral que la Arquitecta Ángela Barrios Padura, bajo la dirección de los profesores D. Alfonso Jiménez Martín y D. Jesús Barrios Sevilla, de la Escuela Técnica Superior de Arquitectura de Sevilla, 
está realizando sobre las cimentaciones romanas, musulmanas y góticas en la provincia de Sevilla

Por otra parte, algunos de los treinta y dos pilares exentos y ochavados de unos diecisiete metros de altura, que sostienen las setenta bóvedas ojivales que conforman la cubierta, presentan fisuras de diversa entidad que aparecen a la altura de 2-3 m desde la base y terminan a los 5-6 $\mathrm{m}$. Con este estudio se pretende determinar si su origen corresponde a las características geotécnicas del terreno, o a la tipología de la cimentación emplearla en estos pilares, o a posibles alteraciones de los materiales que la conforman.

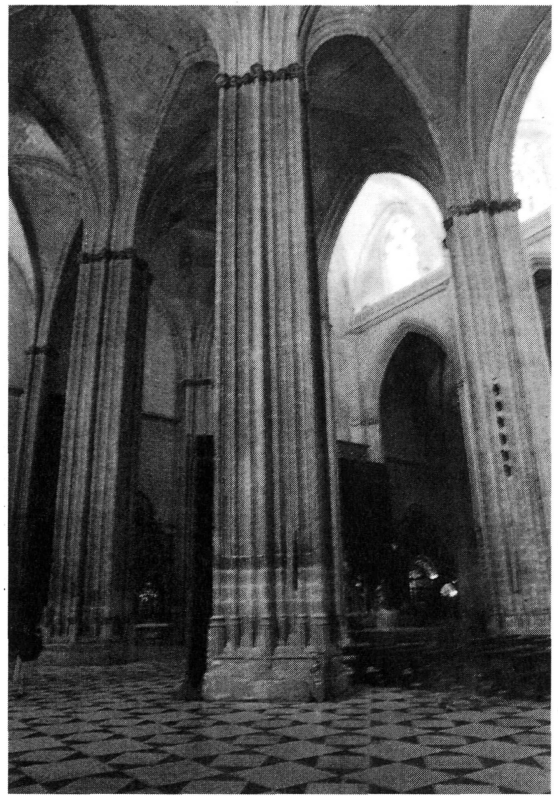

Catedral de Sevilla. Interior

\section{Trabajos desarrollados}

Se han realizado dos sondeos rotatorios, uno en cada uno de los dos pilares elegidos, como representativos de las cargas que soportan y de las fisuras que presentan. Estas perforaciones fueron realizadas por VORSEVI; la primera, separada un metro del pilar, haciéndose la segunda con una inclinación de $19^{\circ}-6^{\prime}$ con respecto a la vertical, con objeto de alcanzar el núcleo de la cimentación y el terreno de apoyo.

Las muestras de testigo continuo obtenidas, tanto de la cimentación como del terreno, han sido analizados y ensayados para, así, poder definir con exactitud sus características.

Por otra parte, el equipo dirigido por el Profesor D. Francisco Borja Barrera ha realizado un detallado estudio geo-arqueológico de las muestras, para datar los yacimientos arqueológicos existentes y el nivel ocupacional de esta zona de Sevilla, que estaba sin caracterizar.
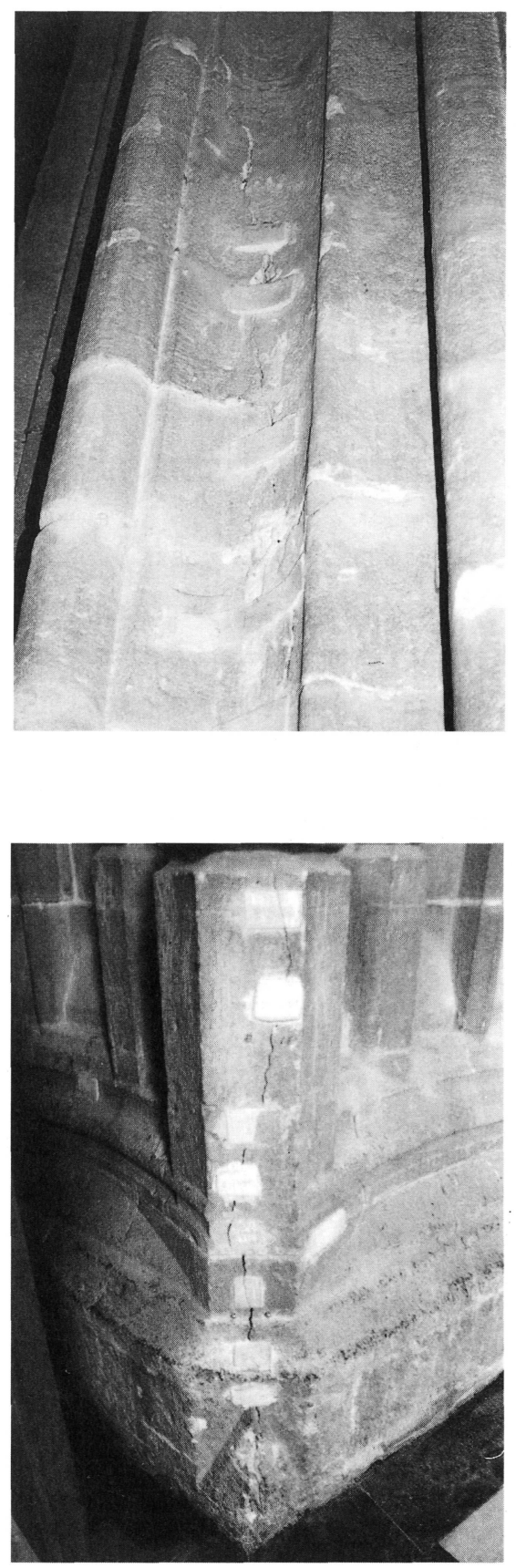

Fisuras en los pilares.

\section{Marco geológico - geotécnico de la ciudad de Sevilla}

La ciudad de Sevilla se enclava en el centro de una amplia llanura fluvial conformada por el río Guadalquivir, cuyos bordes presentan una acusada disimetría, originada por la diferente constitución geológica de ambas márgenes.

El borde Oeste del Aljarafe limita, de forma brusca, con la llanura aluvial del río, por medio de un escarpe rectilíneo de entre 60 y $100 \mathrm{~m}$ de desnivel, constituido por materiales de edad Terciaria (Plioceno-Mioceno), margas, limos y arenas. 
El borde Este de la llanura, de topografia más suave y de relieve relativamente más elevado (Los Alcores), se alinea en la dirección NE-SO, desde Carmona a Dos Hermanas y está constituido preferentemente por arenas calcáreas cementadas (albero)

Volviendo al centro de la llanura que ocupa la ciudad, el río discurre por la misma con un trazado meandriforme que cruza la ciudad de Norte a Sur, recibiendo los afluentes de los arroyos Rivera de Huelva, Tagarete, Tamarguillo y Guadaira, cuyos cauces antiguos han desaparecido, habiéndose modificado algunos de ellos.

El carácter divagante del río, dio lugar en el pasado a la formación de meandros o de cortas naturales (Paleocauces antiguos) que discurren por zonas céntricas de la actual ciudad.

Estableciendo un perfil representativo medio del subsuelo bajo la ciudad, situaríamos en la zona más baja a las denominadas "Margas Azules", del Mioceno, cuyo techo se localiza a profundidades oscilables entre los 5 y $6 \mathrm{~m}$ en la zona Norte, y los $25 \mathrm{~m}$ en el sector CentralOeste.

Por encima de las Margas Azules se sitúan sedimentos cuaternarios de origen fluvial, integrados por gravas arenosas (zahorras), situadas inmediatamente encima de las Margas y de compacidad, en general, elevada. A las gravas se superponen un conjunto de arenas limosas y limos arenosos de hasta $10 \mathrm{~m}$ de espesor.

Finalmente, los suelos más superficiales corresponden a arcillas de tonalidades mayoritarias marrones, a veces grisáceas, de media a baja consistencia.

El espesor de los distintos conjuntos es muy variable de unos sectores a otros de la ciudad, por el propio origen fluvial de dichos depósitos.

Ligado al río Guadalquivir se desarrolla un nivel freático bajo toda la ciudad, cuya profundidad es variable de unos puntos a otros, en función de la cota topográfica y de condicionantes locales, pudiendo oscilar entre los 2 - $3 \mathrm{~m}$ hasta los $9 \mathrm{~m}$, susceptible de variar con las oscilaciones del río.
También hay que indicar la frecuente formación de niveles freáticos colgados en los niveles arcillosos superiores por roturas de tuberías o saneamientos, que no deben de confundirse con el principal del río.

\section{Niveles estratigráficos diferenciados. Caracterización geomecánica}

Los dos sondeos efectuados ponen de relieve la existencia de un importante desarrollo en la cimentación de los pilares, que se extiende hasta los $5,70 \mathrm{~m}$ en $\mathrm{S}-1$ y hasta los 5,90 metros en $\mathrm{S}-2$, una vez realizada la corrección, por inclinación, del sondeo (S-2).

Por debajo de la cimentación hemos distinguido un nivel blando, constituido por suelos aluviales recientes de Arcillas limosas o Arenas arcillolimosas marrones y grisáceas, con restos cerámicos dispersos en su seno. Este nivel tiene un desarrollo de 7,35/8,10 $\mathrm{m}$ y da paso, finalmente, a los $13,25 / 13,80 \mathrm{~m}$ a los niveles granulares densos de arenas limosas con gravas.

El nivel freático se sitúa a 3,70 m de profundidad y está sujeto a variaciones propias del régimen hídrico existente y de las condiciones hidrogeológicas del subsuelo.

A continuación, vamos a estudiar más detenidamente los niveles diferenciados en el subsuelo, comenzando desde las zonas superficiales.

\section{NIVEL 1: Solería y cimentación de las pilastras}

Se reconoce en ambos sondeos en posición superior con el desarrollo indicado en la Tabla 1 .

En estos espesores incluimos a la solería o losa superior de mármol blanquecino o gris $(7 / 8 \mathrm{~cm})$, y parte de la arenisca de la pilastra cortada en el sondeo $\mathrm{S}-2$, inclinado entre 0,09 y $0,40 \mathrm{~m}$.

En el apartado 5 se efectúa un análisis más exhaustivo sobre la composición de la cimentación y sus características físico-químicas y resistentes.

Tabla 1

(1,


Tabla 2

\begin{tabular}{|c|c|c|c|}
\hline 1.1111 .1 & : & : : & $1 \times 14,8 \% 1411$ \\
\hline 1,1 & $5.1 \%$ & 13,00 & $1,1 /$ \\
\hline $18 \%$ & $1, .11$ & 121 & $1,1 \%$ \\
\hline
\end{tabular}

Tabla 3

\begin{tabular}{|c|c|c|}
\hline & : & : \\
\hline 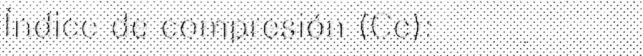 & 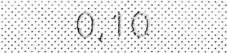 & 1,13 \\
\hline 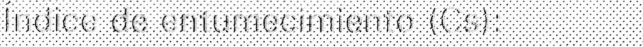 & 011 & $0,1 \%$ \\
\hline 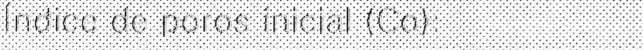 & 103 & 018 \% \\
\hline 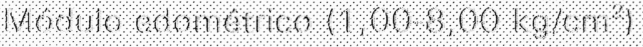 & $1 \%$ & 111 \\
\hline 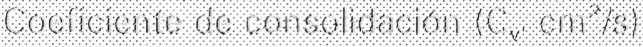 & & \\
\hline & 1, - & 0,16 \\
\hline
\end{tabular}

No obstante, diremos que se trata de una argamasa alternando con hiladas de ladrillo de competencia moderada, con valores de resistencia a compresión simple, que oscilan entre 43,1 y $62,8 \mathrm{~kg} / \mathrm{cm}^{2}$.

NIVEL 2: Aluvial reciente: Arcillas limosas y arenas arcillolimosas de coloraciones marrón grisáceas oscuras, con restos cerámicos dispersos.

En el nivel sobre el que apoyan las cimentaciones reconocidas, apareciendo con la acotación que puede observarse en la Tabla 2.

\section{Composición}

Los numerosos ensayos realizados sobre esta capa nos muestran materiales un tanto diversos en su granulometría, arcillas arenosas y arenas con fracción limoarcillosa, en intercalaciones irregulares (CL, SM-SC, GC-GM).

Predominan las tonalidades grisáceas oscuras o marrón grisáceas, y gris verdosas a la base, siendo frecuente, además, el reconocimiento de restos cerámicos dispersos a cotas muy desiguales. Se trata, pues, de un depósito aluvial relativamente reciente.

El porcentaje de fracción fina oscila entre el 33 y $99 \%$.

El porcentaje de fracción arena oscila entre el 1 y el $33 \%$.
El porcentaje de grava o restos cerámicos oscila entre el 0 y el $44 \%$.

La plasticidad aumenta con la profundidad, con límites líquidos (LL) de 28,1/24,5 a 43,4/45,3.

El índice de plasticidad oscila entre 4,8 y 8,0, y 18,3/22,2.

Deformabilidad. Cambios de volumen

Al tratarse de suelos permanentemente saturados, por situarse bajo el nivel freático, están obviadas las problemáticas relativas a expansividad y colapso.

Los ensayos edométricos realizados han puestode manifiesto valores altos de deformabilidad, obteniéndose los parámetros indicados en la Tabla 3.

\section{Parámetros resistentes}

Los ensayos de compresión simple sobre las muestras inalteradas obtenidas, muestran valores bajos en las cotas superiores de $q_{u}=0,19 / 0,29 \mathrm{~kg} / \mathrm{cm}^{2}$ a $6,00 / 6,58 \mathrm{~m}$, debido a la menor consistencia y a la abundancia de fracción arenosa, que dota a este suelo de un comportamiento no estrictamente cohesivo.

En los testigos parafinados obtenidos: sobre los $11 \mathrm{~m}$ de profundidad, el valor de $q_{u}$ es mayor, oscilando entre 0,61 y $0,99 \mathrm{~kg} / \mathrm{cm}^{2}$, representativos de un suelo cohesivo, 


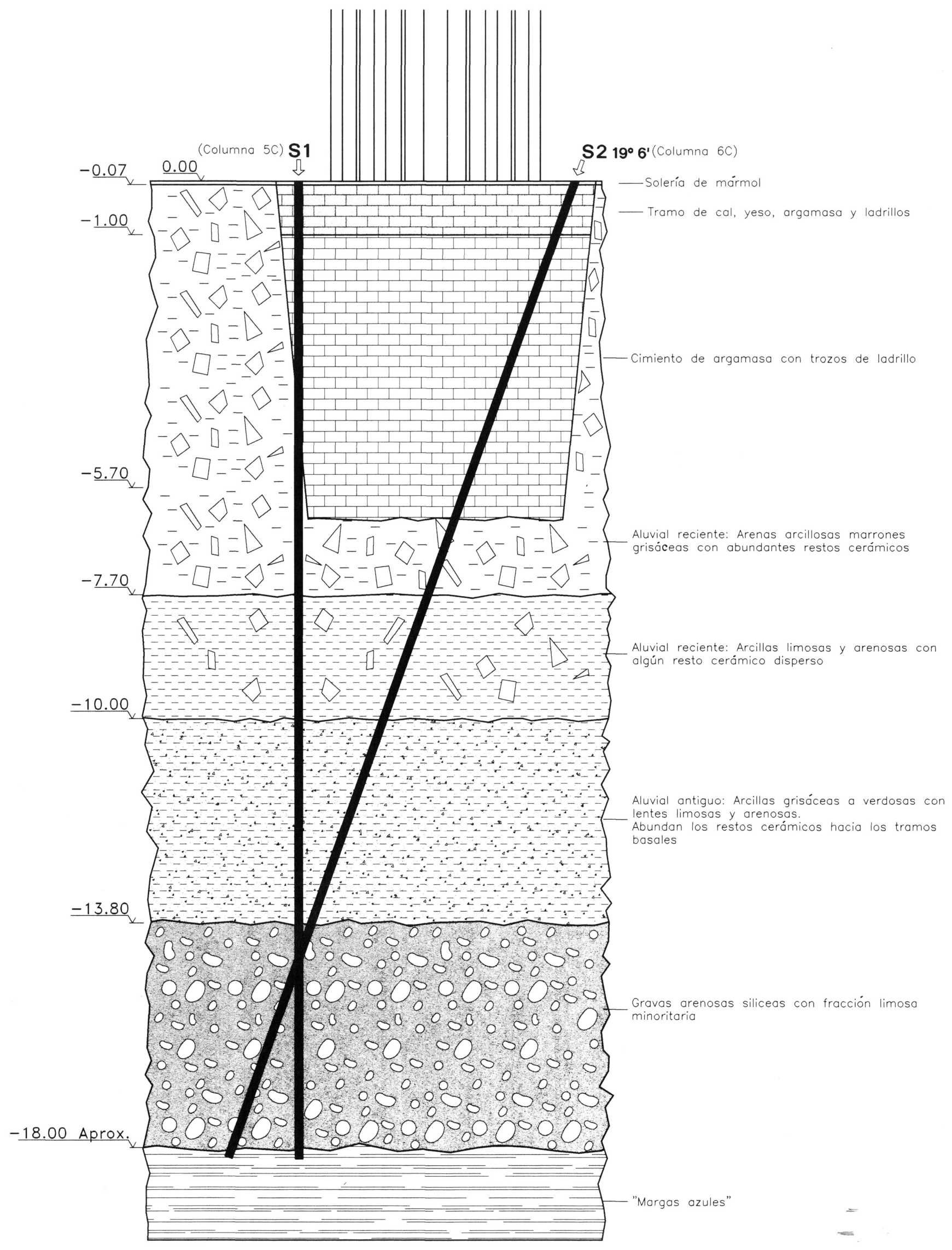

Reconocimiento geotécnico y de cimentación de pilastras en la catedral de Sevilla. 
correspondiéndole un valor de resistencia al corte sin drenaje $(\mathrm{Cu})$ de 0,30 y $0,49 \mathrm{~kg} / \mathrm{cm}^{2}$.

Para determinar los parámetros resistentes a largo plazo, se han realizado ensayos de corte directo consolidados y drenados, obteniéndose los siguientes valores:

Cohesión $C^{\prime}=$ Variable entre 0,03 y $0,17 \mathrm{~kg} / \mathrm{cm}^{2}$

Ángulo de rozamiento interno $\phi '=$ Variable entre 19,4 y $25,9^{\circ}$

Los SPT en el sondeo realizado justo bajo la cimentación, arrojaron un valor de $\mathrm{N}=10$ ( $\mathrm{S}-1$ a $6,60 \mathrm{~m}$ ), compacidad suelta (suelo granular-cohesivo). En el sondeo S-2 se realizó otro SPT, de valor $\mathrm{N}=4$, considerado muy bajo; $\sin$ embargo, este valor no tiene la representatividad del anterior, al realizarse la perforación ligeramente inclinada.

\section{NIVEL 3: Arenas con gravas}

Es el nivel granular grueso, típico de la base del depósito aluvial del subsuelo de Sevilla.

Aparece en ambos sondeos, a las cotas indicadas en la Tabla 4.

Es un suelo del tipo GP, mezcla de gravas y arenas, con escasa fracción limosa, de tonalidad marrón a gris. A la profundidad de 18,40 - 18,55 comienza la marga azul.

Por los datos obtenidos de anteriores estudios, es el nivel de mayor compacidad, en el que se consiguen elevados golpes en los ensayos de penetración, culminando en rechazo.

Parámetros:

Clasificación

GP
$\%$ Para tamiz n ${ }^{\circ} 4$ ASTM

$45 / 62$

$\%$ Para tamiz n ${ }^{\circ}$ 200ASTM

$1 / 6$

Cohesión (C')

0

Ángulo de rozamiento interno ( $\left.\phi^{\prime}\right) \quad 33-36^{\circ}$

\section{Análisis y ensayos a muestras de cimentación}

De los testigos extraídos en los sondeos efectuados se han elegido, como representativos del conjunto, los siguientes:

\section{Sondeo 1}

M-1.- De 1,00 a 1,20 m de profundidad. Corresponde a una argamasa de cal, con ladrillos cerámicos de $6 \mathrm{~cm}$ de espesor.

M-2.-De 3,05 a 3,70 $\mathrm{m}$ de profundidad. Corresponde a una argamasa de cal, con ladrillos cerámicos de $6 \mathrm{~cm}$ de espesor.

M-3.-De 5,00 a 5,20 $\mathrm{m}$ de profundidad. Corresponde a una argamasa de cal, con ladrillos cerámicos de $6 \mathrm{~cm}$ de espesor.

Sondeo 2

M-4.- De 0 a $0.40 \mathrm{~m}$ de profundidad. Roca arenisca.

M-5.- De 1,60 a 1,75 m. Argamasa de cal, con ladrillos cerámicos de $6 \mathrm{~cm}$ de espesor.

M-6.- De 2,70 a $3 \mathrm{~m}$. Argamasa de cal, con ladrillos cerámicos de $6 \mathrm{~cm}$ de espesor.

M-7.- De 5,20 a 5,50 m. Argamasa de cal, con ladrillos cerámicos de $6 \mathrm{~cm}$ de espesor.

Tabla 4 


\section{RESULTADOS}

Características físicas y mecánicas.

\begin{tabular}{|c|c|c|c|c|}
\hline 171.1 .417 .7 & : & 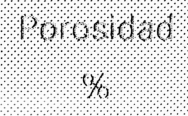 & 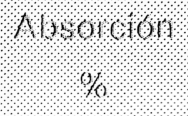 & 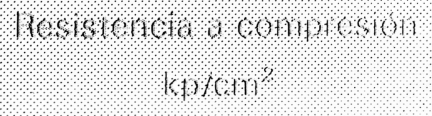 \\
\hline l: & $1 / 1.1 /$. & 1.6 .1 & 1/. & 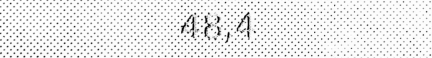 \\
\hline 1.1 & 1.10 .0 & V. & $6 / v^{2}$ & $1 / 6 \cdot 10$ \\
\hline . & $1.1 .1 /$. & V. & $16^{1}$ & 1.0 .8 .8 \\
\hline (1) & 1.1 .1 .6 & 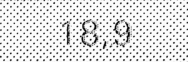 & .19 .6 & 10.8 .0 \\
\hline . & 1.1 .1 .1$. & 1.0 .1 .6 & $.10 .1 \%$ & 6.1 .6 \\
\hline (1) & .1 .14$. & (.) & $.26 .1 \%$ & (1).:. \\
\hline $1.1 \%$ & 1.16 & 1. & \%.: & (1). \\
\hline
\end{tabular}

Composición química

\begin{tabular}{|c|c|c|c|c|c|c|}
\hline $41,401 / 4 \%$ & ': & 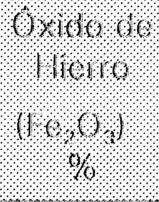 & 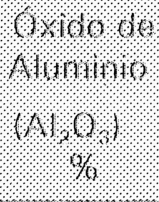 & :1: & 1/4: & : \\
\hline 4 & $1.1 .0 \%$ & 1,1 & 1.1 .10 & 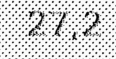 & 1.8 .8 & $1.1 \%: 13$ \\
\hline ? & 1.7 .0 & $.1 .1 \%$ & $1.6 \%$ & 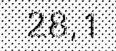 & .0 .0 & $.2 .1 .1 \%$ \\
\hline (1) & $1 \% 1 \%$ & . & $1.1 .1 \%$ & .19.1.5. & (1.) & 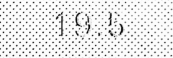 \\
\hline 19 & (10.6. & . & 1.1 .6 & 1.1.1. & 8.6 & 1.1 .9 .1 \\
\hline 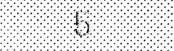 & 1.1 .6 & $1.1 \%$ & $1.1 \%$ & .8 .10 .10 & 0.0 & 1.7 .90 \\
\hline 98 & . 1.0. & $1.1 \%$ & 1.3 & $101.1 \%$ & $1.1 \%$ & 18.0 \\
\hline 1. & 10.2 & .1 .8 & (1.1.5. & $46.1 \%$ & 6.10 & $11 \%$ \\
\hline
\end{tabular}

Compuestos químicos agresivos

\begin{tabular}{|c|c|c|c|c|c|}
\hline 1/11 & \%:14: & (1.7. & 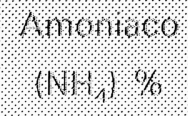 & 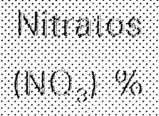 & 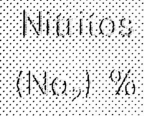 \\
\hline 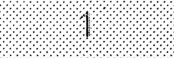 & 1.6 & $6.1 \%$ & 1.1 .1 .1 .8$. & 6.10. & $11.17,11.9$ \\
\hline , & 1.0 & $1.0 \%$ & 1.1 .0 .8 .6$. & 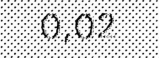 & 1.47 .140 \\
\hline .: & 1.0 .10 & 1.106 & $1.17 .6 .1 \%$ & 1.1 .1 .4 & 17.7 .8 .8 \\
\hline 1) & 1.1 .9 & $9.1 .1 \%$ & 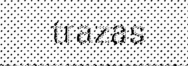 & (1.0.6. & 1.10 .8 .9 \\
\hline (.) & .8 .0 & (1): & 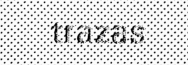 & 1.070 & .1 .1 .0 .0$. \\
\hline 6 & (1.) & $1.0 .11 \%$ & (1: & (. .1 .1 & 1.1 .4 .1 .6 \\
\hline 1 & 1.0 .0 & .0 .1 .18 & 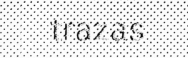 & 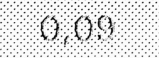 & 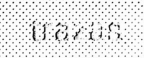 \\
\hline
\end{tabular}




\section{OTRAS DETERMINACIONES}

- Se comprobó la relación en peso entre la fracción de mortero o argamasa de cal y la de ladrillo. El resultado ha sido:

$* 55 \%$ en peso de mortero

$* 45 \%$ en peso de ladrillo

- La argamasa o mortero calizo se ha atacado con ácido, con objeto de comprobar la naturaleza de los áridos. Puede decirse que:

* La arena es silícea, de grano anguloso, con presencia de anfibolitas y esquistos, feldespatos y micas.

* El conglomerante es calizo, de tamaño inferior a $0,2 \mathrm{~mm}$. Está perfectamente carbonatado y su compacidad es variable.

\section{Cálculos geotécnicos}

\subsection{Carga de hundimiento de la cimentación}

Con los datos obtenidos en el reconocimientogeotécnico de los ensayos del sector de los pilares, va a efectuarse una estimación de la carga de hundimiento de la cimentación realizada, considerando que la misma es maciza y que sobresale en anchura, del perímetro del pilar, un metro.

\section{DATOS PREVIOS SELECCIÓN DE PARÁMETROS GEOTÉCNICOS}

Profundidad de cimentación $5,80 \mathrm{~m}$

Dimensiones en planta de las

cimentaciones de las pilastras

$4,30 \times 4,30 m$

Resultado de un sobreancho de $1 \mathrm{~m}$ con respecto a las dimensiones de las columnas.

\section{ESQUEMA GEOTÉCNICO DE CÁLCULO SIMPLIFICADO (SONDEO S-1 Y S-2)}

De 0,00 a 5,70/5,90. Cimiento de hormigón. Profundidad media $=5,80 \mathrm{~m}$.

De 5,70/5,90 a 13,25/13,80 m. Aluvial reciente: Arenas arcillolimosas y arcillas marrones grisáceas (SC-SM, CL)

Cohesión $\mathrm{C}^{\prime}=0,11 \mathrm{~kg} / \mathrm{cm}^{2}$

Ángulo de rozamiento interno $\phi^{\prime}=22^{\circ}$

Densidad aparente $\gamma_{\mathrm{ap}}=1,79 \mathrm{t} / \mathrm{m}^{3}$

Densidad sumergida $\gamma^{\prime}=0,79 \mathrm{t} / \mathrm{m}^{3}$

De $13,25 / 13,80 \mathrm{~m}$ a $17,00 \mathrm{~m}$ (mínimo). Gravas arenosas (GP).
Cohesión $C^{\prime}=0 \mathrm{~kg} / \mathrm{cm}^{2}$

Ángulo de rozamiento interno $\phi^{\prime}=33-36^{\circ}$

Densidad aparente $\gamma_{\mathrm{ap}}=2,1 \mathrm{t} / \mathrm{m}^{3}$

Densidad sumergida $\gamma^{\prime}=1,1 \mathrm{t} / \mathrm{m}^{3}$

NIVEL FREÁTICO $=-3,70 \mathrm{~m}$

\section{CÁlCULO ANALÍTICO DE LA CARGA DE HUNDIMIENTO DE LOS PILARES}

Se aplica la conocida fórmula de Brinch-Hansen, en la cual la componente vertical que produce el hundimiento viene dada por la expresión:

$$
\mathrm{P}_{\mathrm{vh}}=\mathrm{qN}_{\mathrm{q}} \mathrm{S}_{\mathrm{q}} \mathrm{i}_{\mathrm{q}}+\mathrm{CN}_{\mathrm{c}} \mathrm{S}_{\mathrm{c}} \mathrm{i}_{\mathrm{c}}+0,3 \mathrm{~B}_{\gamma} \mathrm{N}_{\gamma} \mathrm{S}_{\gamma} \mathrm{i}_{\gamma}
$$

Donde:

$q=$ Sobrecarga de tierras a la profundidad de cimentación, en el entorno del cimiento

$\mathrm{c}=$ Cohesión

$\gamma=$ Peso específico del suelo

$\mathrm{N}_{\mathrm{q}}, \mathrm{N}_{\mathrm{c}}$ y $\mathrm{N}_{\mathrm{\gamma}}=$ Coeficientes de capacidad de carga, dependientes del ángulo de rozamiento del terreno.

$\mathrm{S}_{\mathrm{q}}, \mathrm{S}_{\mathrm{c}}, \mathrm{S}_{\gamma}=$ Coeficiente de forma

$i_{g}, i_{c}, i_{\gamma}=$ Coeficiente de inclinación

El terreno de empotramiento del cimiento se asimila al de apoyo.

$$
\mathrm{P}_{\mathrm{vh}}=122,12 \mathrm{t} / \mathrm{m}^{2}
$$

Resultandocon unas dimensiones en plantas del cimiento de $4,30 \times 4,30 ; P_{v b}=2.287 \mathrm{t}$ por pilar.

\section{COEFICIENTE DE SEGURIDAD ANTE EL HUNDIMIENTO}

Viene regido por el cociente entre la carga de hundimiento de la cimentación y la tensión realmente transmitida:

$$
\mathrm{F}=\frac{\mathrm{P}_{\mathrm{v}} \text { hundimiento }}{\mathrm{P} \text { transmitida }}
$$

\section{CARGAS TRANSMITIDAS}

En este apartado se ha considerado la parte correspondiente de bóvedas y cubiertas, el pese del pilar y el de la cimentación. Los datos de composición y densidad son estimativos, aunque están tomados de otros estudios 
realizados en diferentes zonas de la catedral. Con estos datos resulta que las cargas que se transmiten son, aproximadamente, las siguientes:

$$
\begin{array}{cc}
\text { Pilar 5 C } & 570 \mathrm{t} \\
\text { Pilar 4 C } & 720 \mathrm{t} \\
\text { El factor de seguridad ante el hundimiento quedaría así: } \\
\text { PILAR 5C } & \mathrm{F}=2257 / 570=3,95 \\
\text { PILAR 4C } & \mathrm{F}=2257 / 720=3,13
\end{array}
$$

Ambos factores están cercanos al mínimo de 3 exigido y pueden considerarse aceptables para garantizar la estabilidad a largo plazo del terreno de asiento del cimiento.

\subsection{Estimación de asientos}

Puede efectuarse una estimación de los asientos acontecidos de acuerdo con los parámetros obtenidos en el reconocimiento.

Utilizamos para ello el método edométrico.

$$
\mathrm{S}=\mathrm{H} / 1+\mathrm{e}_{\mathrm{o}} \mathrm{C}_{\mathrm{c}} \log \left(\frac{\delta_{\mathrm{io}+\Delta \delta \mathrm{i}}}{\delta_{\mathrm{io}}}\right)
$$

$\mathrm{C}_{\mathrm{c}}=$ Índice de compresión

$\mathrm{H}=$ espesor capa compresible

$\delta_{i o}=$ Tensión inicial a cota de cimentación

$\Delta \delta=$ Incremento de tensión inducido por la cimentación

\section{Datos previos}

- Nivel de cimentación medio: $-5,80 \mathrm{~m}$

- Nivel de base suelos blandos arcillosos: $-13,80 \mathrm{~m}$

- Espesor suelos blandos compresibles H: 8,00 m

- Índice de compresión $\mathrm{C}_{\mathrm{c}}=0,13$

- Índice de poros $\mathrm{e}_{0}=0,853$ $-\delta_{\text {io }}=8,09 \mathrm{Tm} / \mathrm{m}^{2}$

$-\Delta \delta=$ Variable entre 30,88 y $38,96 \mathrm{t} / \mathrm{m}^{2}$

- Escogemos el valor más alto a efectos de cálculo.

Estimación de asientos absolutos:

$$
\mathrm{S}=42,65 \mathrm{~cm}
$$

Como podemos apreciar los asientos totales son altos $(>42,65 \mathrm{~cm})$ en función de las cargas transmitidas. Sin embargo, el largo período de construcción de la catedral contribuyó a una consolidación y drenaje progresiva, por lo que queda garantizada la estabilidad.

\section{Tiempo de asentamiento (Tabla 5)}

Suponiendo al terreno compresible homogéneo, el tiempo de asentamiento viene dado por:

$$
\mathrm{T}=\frac{\mathrm{T}+\mathrm{H}_{\mathrm{d}}^{2}}{\mathrm{C}_{\mathrm{v}}}
$$

Siendo:

$\mathrm{T}=$ Factor de tiempo adimensional, calculado por la teoría en función del grado de consolidación $\mathrm{V}$, o porcentaje del asiento $S$ que se desee considerar.

$\mathrm{H}_{\mathrm{d}}=$ Espesor del terreno que drena hacia las superficies permeables existentes.

$\mathrm{C}_{\mathrm{v}}=$ Coeficiente de consolidación, deducido de la curva asientos tiempo, del ensayo edométrico para el escalón de carga correspondiente.

$$
\mathrm{H}_{\mathrm{d}}=4,00 \mathrm{~m} ; \mathrm{C}_{\mathrm{v}}=4,2 \times 10^{-4} \mathrm{~cm}^{2} / \mathrm{s}
$$

\begin{tabular}{|c|c|c|}
\hline 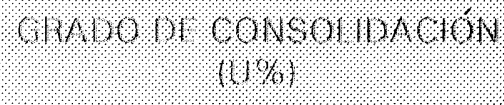 & ', & (1) \\
\hline $10 \%$ & 4,26 & 36, ? dias \\
\hline $30 \%$ & 12,79 & $31 /$ das \\
\hline $50 \%$ & 21,32 & 881 dies \\
\hline $70 \%$ & 29.85 & 4 años y 30 ? días \\
\hline $90 \%$ & 38,38 & 10 drios y $31 /$ dadif \\
\hline $98 \%$ & 41,79 & 18 anos y 40 bas \\
\hline $99 \%$ & 42,22 & 24 años y 54 dios: \\
\hline
\end{tabular}

Esta tabla teórica es sólo indicativa, por cuanto que el período de construcción fue de unos noventa años y el ritmo de carga del terreno mucho más lento que el supuesto. Otro dato a tener presente es la homogeneidad del subsuelo y de la construcción, lo que implica la ausencia de asientos diferenciales, como puede constatarse.

Tabla 5 


\section{Comentarios}

- La cimentación de las columnas tiene un sobreancho de $1 \mathrm{~m}$ y una profundidad de 5,70 $-5,90 \mathrm{~m}$.

- La constitución de esta cimentación es una argamasa de cal, con intercalaciones de ladrillos y algunas gravas sueltas, de forma que su composición media es:

$-55 \%$ en peso de mortero de cal; que está constituido por un $50 \%$ de arena silícea de grano anguloso, con presencia de anfibolitas, esquistos, feldespatos y micas y el otro $50 \%$ por cal, con un tamaño inferior a $0,2 \mathrm{~mm}$, que está perfectamente carbonatada.

$-45 \%$ de ladrillos, de $6 \mathrm{~cm}$ de espesor y gravas sueltas de naturaleza silícea.

-Las características físicas y mecánicas de este conglomerado de mortero, ladrillo y gravas son:

\section{- Densidad \\ - Porosidad \\ - Absorción \\ -Resistencia a compresión $\mathrm{kg} / \mathrm{cm}^{2}$}

Oscila entre 1,45 y $1,73 \mathrm{gr} / \mathrm{c} . \mathrm{c}$.

Oscila entre el 18,9 y $31,0 \%$

Oscila entre el 14,4 y el $26,9 \%$

Oscila entre 43,1 y $62,8 \mathrm{~kg} / \mathrm{cm}^{2}$, con un valor medio de $51,7 \mathrm{~kg} / \mathrm{cm}^{2}$

-La composición química corresponde a la de un mortero de cal tipo medio $1: 1$ aproximadamente, conformado por una arena silícea y cal.

-No hay presencia de sulfatos, por lo que la cantera de caliza no contiene piedra de yeso o algez.

-El análisis del agua freática que está en contacto con el cimiento de la columna indica la presencia de nitratos, nitritos y amoniaco, como corresponde a un "agua colgada" procedente de la rotura de canalizaciones y otras, con contenidos de materia orgánica en proceso de descomposición.

-Los resultados de los análisis y ensayos efectuados a la muestra de piedra arenisca que apoya en la cimentación de la columna $4 \mathrm{C}$, son los siguientes:

- Porosidad

- Absorción

- Resistencia a compresión

-El análisis químico indica una relación fracción silícea:caliza de 1:1,75

- Dado que la construcción de pilares y bóvedas de la catedral se tardó en realizar unos 90 años, el terreno de apoyo se fue drenando de una forma lenta, produciéndose la disipación de las presiones intersticiales. Esto es lo que explica el buen comportamiento de la cimentación analizada. Si la construcción se hubiera realizado en un corto período de tiempo, no se habría producido la disipación de las presiones intersticiales de esta forma, por lo que muy probablemente se habría producido el hundimiento y caída de la referida construcción, 0 , en el caso más favorable, una importante inclinación de las mismas.

\section{Resumen del estudio geo-arqueológico}

El estudio arqueológico realizado pone de manifiesto una serie de datos que pueden considerarse de excepcional interés. Se han identificado y descrito tanto los componentes naturales (sedimentos, rasgos edáficos, restos de fauna, etc.) como arqueológicos (cerámicas, restos constructivos, vidrios, huesos, etc.) en las muestras de suelo tomadas en los dos sondeos realizados. Se han aplicado métodos analíticos físico-químicos, tales como granulometrías, contenido en fósforo, localización de especies como gasterópodos, etc.

Como introducción, puede establecerse el siguiente registro geoarqueológico, tomando como base la descripción de las siguientes Formaciones Superficiales Antrópicas (F.S.A.):

I.- De 0 a 5,7 m, los restos que aparecen corresponden a la época medieval; se trata de las solerías, cimientos de las columnas y otros restos arqueológicos. Corresponden a una F.S.A. ocupacional, correspondiente a lo que denominamos una urbanización progresiva.

II.- De 5,7 a $8 \mathrm{~m}$ de profundidad aparecen unos restos que corresponden a una F.S.A. muy antropizada, correspondiendo al estrato de arenas y limos arcillosos fluviales, con lo que se cierra la laguna Romana existente. Corresponde a la época altoimperial Romana.

III.- De 8 a 13 m de profundidad, existen unos depósitos que corresponden a una F.S.A. poco antropizada, en un estrato de arenas y arcillas fluvio-lagunares de una laguna de llanura aluvial Romana del Alto Imperio Romano.

IV.-'De 13 a $14 \mathrm{~m}$ aparecen gravas fluviales en los que hay depósitos de lechos aluviales Romanos, correspondientes a la época Romano republicana.

Con estos antecedentes puede describirse la situación de la zona en la que se dispone la catedral de Sevilla como la siguiente, pasando desde la zona más profunda a la más superficial:

I.- Unidad basal de gravas aluviales que forman parte del fondo de un canal fluvial en una llanura aluvial abierta dominada por uno o varios canales rectilíneos. El espesor de esta capa va desde la cota - $18 \mathrm{~m}$ a la - 12,5 - 13,5 m. En este conjunto caben distinguir dos niveles o conjuntos sedimentarios diferentes: el de los lechosfluviales históricos y la base de gravas correspondientes a las terrazas fluviales. 
En este estrato hay una ausencia casi total de restos antrópicos, que se puede cifrar en la sola aparición de tres trozos de ladrillo a la profundidad de $14-15 \mathrm{~m}$. El estrato corresponde a una serie de gravas y arenas cuarcíticas, con presencia de pizarras, feldespatos y micas. La filiación crono-cultural del material cerámico encontrado en la parte superior del estrato (13-15 m) corresponde a la época Romano republicana, aunque podría estar formándose desde épocas inmediatamente anteriores, de ahí que se hable en el informe de lecho aluvial romano.

II.- Por encima del lecho de gravas descrito anteriormente se dispone una segunda unidad, compuesta, básicamente, por arenas y arcillas de origen lagunar y fluvio-lagunar, que definen un medio de laguna de llanura aluvial en ámbito cercano a la desembocadura, lo que puede estar indicando el paso a un modelo fluvial de carácter meandrinoso, con abandono de canales, etc. El espesor de este nivel va desde los $8,70-8,80 \mathrm{~m}$ a los $12,5-13,5 \mathrm{~m}$. Por la progresiva incorporación de restos antrópicos, que en estos momentos aún no son predominantes, como delata la presencia de gasterópodos de aguas claras corrientes y abundante vegetación $\mathrm{y}$, por los bajos niveles de contenido en fósforo, se puede concluir que esta unidad se denomine como F.S.A. poco antropizada.

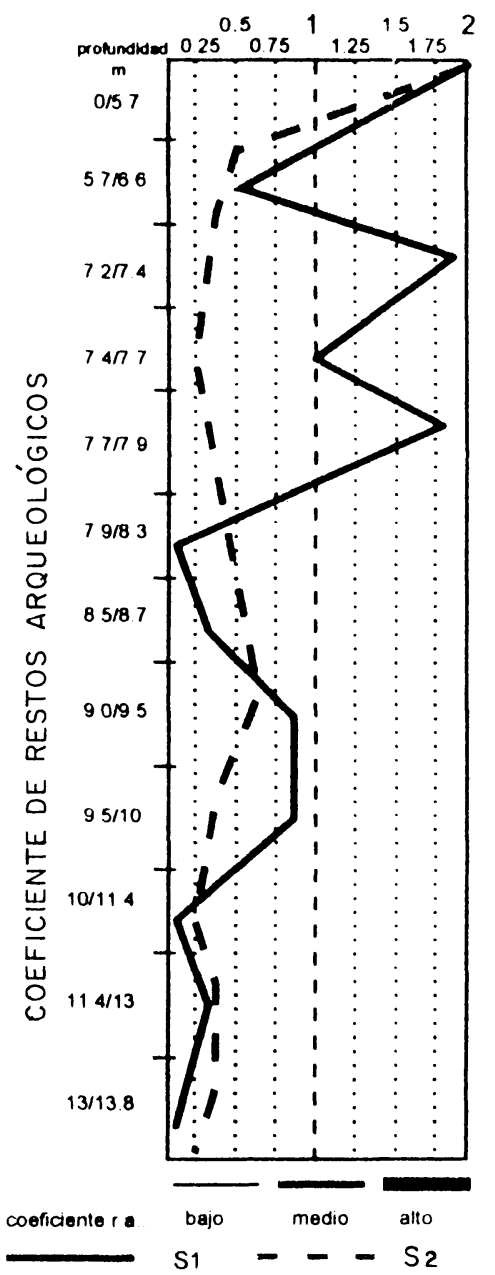

Esta formación tiene su génesis en época Romano altoimperial, justamente cuando se produce la expansión urbana hacia este punto de la Ciudad, el cual queda, según las hipótesis existentes dentro del recinto murado, desde el siglo I DC.

Los restos arqueológicos encontrados corresponden a:

- Restos carbonosos, constructivos y cerámicos, con presencia de restos muy triturados de malacofauna. Igualmente aparecen restos óseos y de escorias. Estos hallazgos corresponden al estrato de 8,70 a $9,50 \mathrm{~m}$

- A partir de 9,50 m, los restos arqueológicos son más abundantes, encontrándose restos óseos, entre ellos un fragmento perteneciente a una costilla de un mamífero de mediano tamaño. Igualmente aparecen gasterópodos del tipo Physa fontinalis, que se desarrolla en aguas claras corrientes y abundante vegetación; escorias de horno, posiblemente cerámico, cerámicas obtenidas a torno con pastas amarillentas, anaranjadas y rojas, apareciendo piezas tales como galbo de "Terra Sigillata" Hispánica, ánforas y cerámica de cocina.

Lacronología correspondeal período Romano altoimperial. 
III.- Corresponde al estrato que aparece entre 7 y $8,70 \mathrm{~m}$ de profundidad, y se trata de un estrato de arenas y limos arcillosos fluviales con presencia de restos arqueológicos. Corresponde a un medio semiconfinado en vías de colmatación, influenciado por aportes de crecidas fluviales y sometido a una fuerte presión antrópica que no llega a consumarse en una ocupación plena del sector. Este tramo corresponde al período Romano altoimperial

Los restos arqueológicos encontrados corresponden a restos óseos, una de cuyas piezas corresponde a un hueso largo de extremidad de ungran herbívoro(vaca, caballoo asno). Igualmente, aparecen restos cerámicos, gasterópodos (animal propio de aguas claras corrientes y abundante vegetación) y restos de escoria. A este respecto cabe destacar el hallazgo encontrado entre 7,40 y $7,70 \mathrm{~m}$, al que se ha determinado el contenido en $\mathrm{P}_{2} \mathrm{O}_{5}$ y cuyos resultados indican una importante presión antrópica y una abundante fauna, encontrándose restos de carbón, malacofauna, restos cerámicos correspondientes a ladrillos, tégula, ánforas y dolia.

Entre los 7,70 y 7,90 m, además de los restos antrópicos identificados anteriormente aparecen restos de escoria de horno de fundición de hierro y restos de cerámica de cocina, ennegrecida por hollín de carbón, tales como cuencos, galbo y ánforas.

El material encontrado entre 8,30 y $8,70 \mathrm{~m}$ es de gran interés, ya que aparece un resto de cuenco de vidrio de tonalidad azul, además de los restos cerámicos hallados a una cota superior.

IV.- Desde la cota - $7 \mathrm{~m}$ hasta la superficie aparecen acúmulos puramente arqueológicos, aunque manteniendo la matriz fina de origen fluvial, cimientos y solerías superpuestas. Estamos ante el tell urbano de la ciudad de Sevilla, que en ese punto presenta una plena ocupación de área, primero en época Romano altoimperial y, posteriormente, con las construcciones almohades y, por último, la catedral. Los fósiles encontrados corresponden a restos óseos correspondientes a costillas de mamífero de mediano tamaño, carbón vegetal, pequeñas láminas de bronce y restos cerámicos.

\section{Conclusiones}

Por los resultados obtenidos en este estudio se deduce que lacimentación existente puede considerarse como adecuada y aceptable. El terreno de apoyo tiene unas deficientes características geomecánicas, pero dado el tiempo empleado en la construcción de las estructuras, columnas, bóvedas y cubiertas (unos 90 años), se consolidó y drenó en los primeros 25 años, por lo que puede soportar las cargas que transmite la actual estructura

Como datos más significativos, se señalan los siguientes:
- La cimentación está constituida por una argamasa de cal, que engloba hiladas de ladrillos macizos de $6 \mathrm{~cm}$ de grueso y gravas, con una buena resistencia a compresión (valor medio $51,7 \mathrm{~kg} / \mathrm{cm}^{2}$ ), superior a la de la piedra arenisca de la columna, que es $32,8 \mathrm{~kg} / \mathrm{cm}^{2}$.

- El canto de la cimentación es de 5,70-5,90 m y el sobreancho de $1 \mathrm{~m}$, lo que pone de manifiesto un adecuado planteamiento. Estos datos coinciden con los obtenidos en el estudio de otras zonas de esta Catedral, por lo que puede deducirse que la cimentación proyectada para todo el conjunto, se ejecutó de forma continuada y con un criteriouniforme de profundidad, materiales y composición.

- El terreno de apoyo de la cimentación ha tenido un buen comportamiento en aquellos elementos constructivos cuyo período de construcción ha sido largo, lo que ha permitido que el terreno se consolidase de forma adecuada, por cuanto que las presiones sobre el suelo se han ido incrementando de forma muy lenta y la disipación de las presiones intersticiales se ha realizado de forma diferida. Consecuencia de ello es que el terreno presenta en la actualidad un coeficiente de seguridad superior a 3 , frente a la carga de hundimiento, por lo que la carga admisible puede considerarse adecuada. Los asientos se han producido de forma lenta y uniforme, sin haberse puesto de manifiesto alteraciones visibles en la estructura. Si la construcción se hubiera realizado de una forma rápida no se hubiera producido la disipación lenta de las presiones intersticiales, originando importantes asientos, con consecuencias tales como desplomes de los elementos construidos. Este puede ser el caso de la Puerta de Campanillas y Cimborrio de esta catedral, cuya construcción se realizó en un corto período de tiempo.

- Descartadoel terreno como posible causa de las fisuraciones que se observan en diferentes pilares de la catedral de Sevilla, queda la necesidad de conocer las características físicas, mecánicas, deformabilidad, estabilidad y otros aspectos de los pilares. Esta exigencia se hace en base a la existencia de fisuras en las columnas, que pueden aumentar con el tiempo y que, tal y como ha quedado demostrado, no tienen una causa u origen geotécnico. Éste será el tema de la próxima investigación.

El trabajo desarrollado por el equipo geoarqueológico dirigido por el profesor Borja, aporta una importante novedad en la reconstrucción paleogeográfica del valle bajo y desembocadura del Guadalquivir. Hasta el presente se conocía la existencia de una terraza fluvial sepultada bajo los rellenos fluviales recientes, que se creían coetáneos con los momentos del Holoceno superior (un milenioa. C.). Igualmente se conocían los rellenos fluviales que hoy dan la topografía básica de nuestra ciudąd. Quedaba, por tanto, sin aclarar el paso de una situación a otra, tanto en lo que se refiere al mecanismo fluvial que lo ejecuta, como desde la cronología del proceso y su relación con las variaciones del nivel del mar. En este estudio estos hechos aparecen 
plenamente interconectados, estableciéndose la siguiente secuencia:

1. Terraza fluvial Holoceno superior. El nivel del mar es alto (1.000 - 750 años a.C.).

2. Incisión fluvial protohistórica sobre terraza fluvial preexistente. El nivel del mar es bajo (de 700 años a.C. a época Romano republicana).
3. Lechos aluviales Romano republicanos. El nivel de mar sigue estando bajo (de 700 años a.C. a época Romano republicana).

4. Relleno lagunar, fluvio-lagunar y antrópico ocupacional. El nivel del mar vuelve a estar alto en fase plenomedieval (800 - 950 años d.C.) y bajada de los niveles marinos hasta las posiciones subactuales.

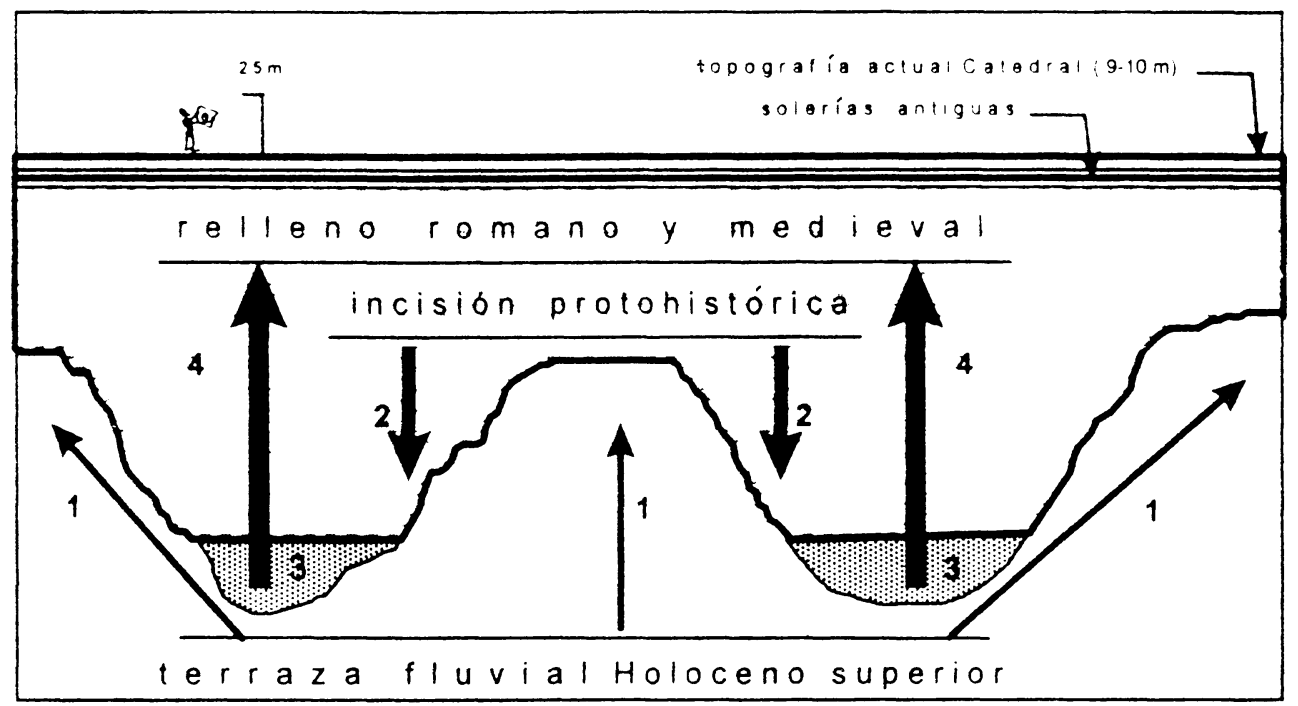

1) Terraza fluvial Holoceno superior/alto nivel del mar en $1.000 / 750$ años a.C. 2) Incisión fluvial protohistórica / bajo nivel del mar en 750 años a.C. (Época Romano republicana. 3) Lechos aluviales Romano republicano/bajo nivel del mar del mar romsno. 4) Relleno lagunar, fluvio-lagunar y ocupacional / alto nivel del mar en fase plenomedieval. ( $800 / 950$ años d.C.) y bajada hasta los niveles subactuales.

Estratigrafía con registro arqueológico romano actual.

Gravas de lecho aluvial romano.

Sintesis de las fases de evolución paleogeográfica del subsuelo de la catedral de Sevilla.

\section{BIBLIOGRAFÍA}

(1) Informe de JOSEPH TIRADO, Maestro Mayor de obras y LORENZO FERNÁNDEZ, Maestro de obras de cantería, dirigido al Cabildo de la Catedral el día 15 de noviembre de 1508. Biblioteca Colombina de la catedral de Sevilla

(2) "Desperfectos en el templo". Informe de JOSÉ GESTOSO.

(3) "Memoria sobre las causas del hundimiento de 1 de agosto de 1888" de ADOLFO FERNÁNDEZ CASANOVA. Biblioteca Colombina de la catedral de Sevilla.

(4) “Geotecnia y cimientos": JIMÉNEZ SALAS, JUSTO ALPAÑÉS Y SERRANO GONZÁLEZ.

(5) Normas UNE, NTE, NCSE-94, NLT y ASTM. 